\title{
Sculpting molecular structures from bilayer graphene and other materials
}

\author{
Laith Algharagholy, Steven W. D. Bailey, Thomas Pope, and Colin J. Lambert \\ Department of Physics, Lancaster University, Lancaster LAI 4YB, United Kingdom
}

(Received 6 July 2012; published 13 August 2012)

\begin{abstract}
We demonstrate a technique for creating unique forms of pure $s p^{2}$-bonded carbon and unprecedented heteromolecules. These new structures, which we refer to as sculpturenes, are formed by sculpting selected shapes from bilayer graphene, heterobilayers, or multilayered materials and allowing the shapes to spontaneously reconstruct. The simplest sculpturene is topologically equivalent to a torus, with dimensions comparable to those of fullerenes. The topology of these new molecular structures is stable against atomic-scale defects. We demonstrate that sculpturenes can form the basic building blocks of hollow, multiconnected structures, with potential applications to nanofluidics and nanoelectronics.
\end{abstract}

DOI: $10.1103 / \mathrm{PhysRevB.86.075427}$

PACS number(s): 61.48.Gh, 81.05.ub, 81.07.-b, 85.65.+h

Although a variety of techniques has been developed for synthesizing fullerenes, ${ }^{1-3}$ carbon nanotubes $(\mathrm{CNTs}),{ }^{4-8}$ and more exotic structures, such as CNT Y junctions, T junctions, toruses, bamboos, buds and horns, ${ }^{4}$ their synthesis is invariably uncontrolled. For example, CNTs grown using arc discharge, laser ablation, or chemical vapor deposition typically possess a mixture of chiralities and the more exotic structures usually appear only by chance. On the other hand, the synthesis of graphene nanoribbons (GNRs) is more deterministic and can be achieved using lithographic, ${ }^{9-11}$ chemical, ${ }^{12-15}$ and sonochemical ${ }^{16,17}$ techniques, unzipping $\mathrm{CNTs}^{18-24}$ and assembling GNRs from chemical precursors. ${ }^{25}$ For example, scanning tunneling microscopy (STM) lithography ${ }^{26}$ can be used to cut GNRs with widths as small as $2.5 \mathrm{~nm}$, with a specified chirality, a specified location, and with their ends contacted to (graphene) electrodes. However, unlike fullerenes and closed CNTs, GNRs are not pure forms of $s p^{2}$-bonded carbon because $s p^{2}$ bonding is disrupted by their edges, which are difficult to control at an atomic scale. ${ }^{24}$ This disruption is undesirable because, as a consequence, for a given electron energy gap, the mobility of GNRs is typically significantly lower than that of CNTs. ${ }^{24-32}$ Efforts to control this disruption have led to rapidly increasing interest in the physics and chemistry of graphene edges and, in particular, edge coalescence (EC) in multilayer structures, through which open edges reconstruct to form closed edges with a higher degree of $s p^{2}$ bonding. Open edges and ECs in bilayer graphene have been imaged by a number of groups. ${ }^{33,34}$ After thermal annealing or Joule heating, open edges are less commonly found and the more frequent ECs are observed to run mainly along achiral (zigzag or armchair) directions of both $A A$-stacked and $A B$-stacked bilayer graphene. One of the technological drivers of GNR science is the desire to realize scalable sub-10-nm electronics. ${ }^{35}$ Here we demonstrate that as the $3 \mathrm{~nm}$ length scale is approached, a transition to a new regime occurs, in which the proximity of one EC to another causes the global reconstruction of whole structures and not simply their edges. This global reconstruction enables the creation of new molecular structures, which cannot be synthesized by any other method. The aim of this paper is to establish the principle of this methodology. We demonstrate that by sculpting selected shapes from bilayer graphene and allowing the shapes to reconstruct globally, unique $s p^{2}$-bonded molecular structures can be formed (which we refer to as sculpturenes) and a variety of multiconnected $s p^{2}$-bonded geometries can be created. This reconstruction is demonstrated using density functional theory and, where appropriate, classical molecular dynamics.

As a first example of such sculpturenes, we demonstrate that sculpted bilayer graphene can reconstruct to form new $s p^{2}$-bonded carbon cages, which are topologically distinct from fullerenes. Our simulations leading to the new structures were performed using the SIESTA implementation of DFT. ${ }^{36} \mathrm{Ab}$ initio structural optimization was performed using the generalized gradient approximation (GGA) with norm-conserving pseudopotentials, double zeta polarized (DZP) basis sets of pseudoatomic orbitals, and the Ceperley-Alder exchange correlation functional with the atomic forces relaxed to $5 \mathrm{meV} / \AA$. When computing the total energies, the counterpoise method ${ }^{37}$ was used to eliminate basis-set errors. As an independent check on the predicted structures, we also used classical molecular dynamics (MD) ${ }^{38}$ to study the time evolution of the sculpturene formation in vacuum and at room temperature, within an $N V T$ ensemble. The Dreiding force field ${ }^{39}$ was used to model the intramolecular carbon-to-carbon interactions, and the short-ranged intermolecular van der Waals forces are calculated using the Lennard-Jones potential. A cutoff distance of $8.5 \AA$ was used, with a time step of $1 \mathrm{fs}$, and the total simulation time was typically $1000 \mathrm{ps}$.

Consider cutting a hexagonal shape in bilayer graphene (located on top of a graphene substrate), using, for example, STM lithography, ${ }^{29}$ to form the $A B$-stacked bilayer annulus, shown in Fig. 1(a), where atoms of the upper (lower) layer of the nanoribbon are shown in red (blue) and atoms belonging to the graphene substrate are shaded gray. Figures 1(b) and 1(c) show two views of the resulting toroidal sculpturene, which is formed after allowing this ribbon to relax and spontaneously reconstruct (for clarity, the substrate is no longer shown).

Unlike other pure forms of $s p^{2}$ carbon, such as fullerenes and closed carbon nanotubes, which are topologically equivalent to a sphere and characterized by an order of connection $k=1$, the above toroidal sculpturene possesses a connection of $k=3$. (The order of connection $k$ is 1 plus the number of closed cuts that can be made on a given surface without breaking it apart into two pieces. $)^{40}$ Since the connection $k$ is a topological invariant, toroidal sculpturenes cannot be 


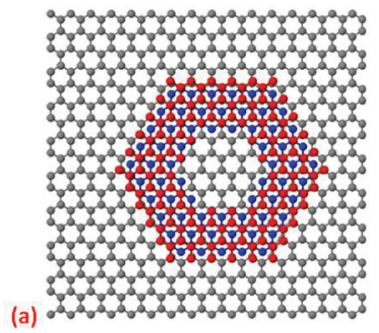

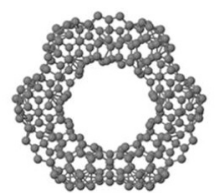

(b)

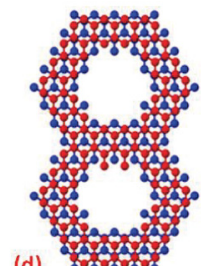

(d)

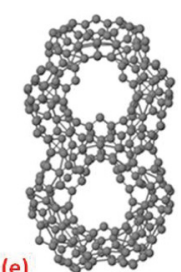

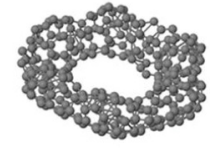

(c)

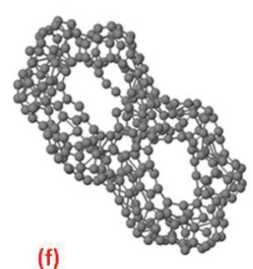

(f)

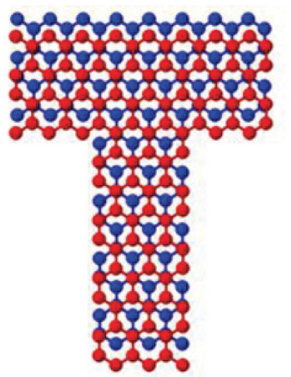

(a)

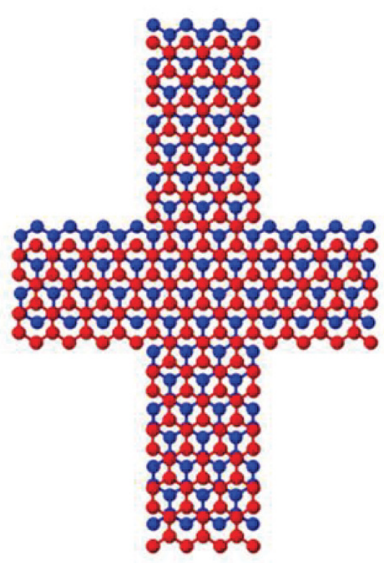

(c)
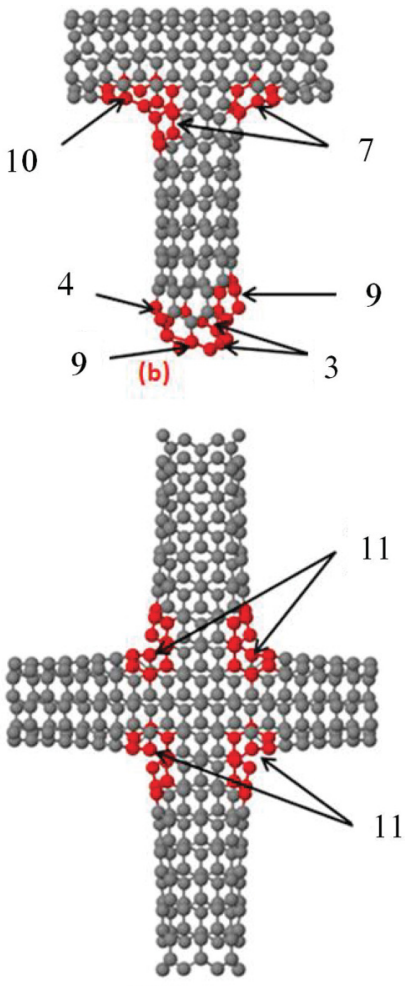

(d) graphene. (e), (f) Different views of the resulting sculpturene with connection $k=5$, obtained by allowing the initial shape to reconstruct.

continuously deformed into fullerenes or nanotubes. Furthermore, since $k$ is unchanged by the introduction of atomicscale defects and impurities, this method of constructing new topologies is extremely robust. In contrast, with conventional fullerenes, the $s p^{2}$-bonded torus shown in Figs. 1(b) and 1(c) is expected to exhibit interesting orbital magnetic effects, including persistent currents, ${ }^{41-46}$ which are a direct consequence of its topology. Furthermore, the presence of a hydrophobic pocket in the center of the torus provides a unique binding site for supramolecular attachment to other molecules. The toroidal sculpturene of Figs. 1(b) and 1(c) is the simplest example of a hierarchy of $s p^{2}$-bonded toroidal molecular structures with higher connections. As a second example, Fig. 1(d) shows a sculpturene formed from two hexagons, which reconstructs to form the "figure of eight" shown in Figs. 1(e) and 1(f), which possesses a connection $k=5$. The toroidal sculpturene of Figs. 1(b) and 1(c) is of the order of 100 times smaller than ring structures reported elsewhere in the literature, which are either formed accidentally ${ }^{47,48}$ or by etching CNTs to form
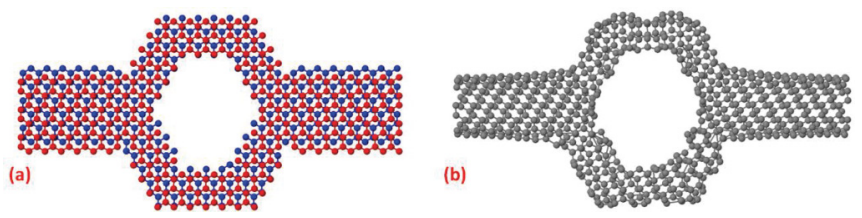

FIG. 2. (Color online) Formation of a hollow torus connected to CNTs. A supercell of $A B$-stacked BiGNR, which contains 676 carbon atoms, (a) before and (b) after reconstruction.
FIG. 3. (Color online) (a) A T-branched structure made by sculpting an $A B$-stacked bilayer. The structure is periodic in the horizontal direction. The corners of the $\mathrm{T}$ shape contain either sevenfold or tenfold rings, as indicated in the figure. The bottom of the $\mathrm{T}$ contains fourfold, threefold, and ninefold rings. (b) The resulting T-branched CNT structure obtained by relaxing the bilayer ribbon of (a). (c) A crosslike structure made by sculpting an $A B$-stacked bilayer. The structure is periodic in the horizontal and vertical direction. (d) A relaxed crosslike carbon nanotube structure obtained by relaxing the bilayer ribbon in (c). The corners contain 11 -fold rings.

loops with diameters of the order of 0.5 microns. ${ }^{49}$ Toroidal molecular structures with $k$ greater than 3 , such as that shown in Figs. 1(e) and 1(f), are unprecedented and cannot be created by any other method.

The structures of Fig. 1 can be regarded as basic building blocks from which more complex interconnected structures can be formed. As an example, Fig. 2(a) shows an initial shape sculpted from bilayer graphene. Upon relaxation, this forms the structure in Fig. 2(b), which comprises a horizontal nanotube (on the left), which bifurcates into two parallel channels. These channels later rejoin and attach seamlessly to a nanotube on the right.

As further examples of hollow interconnects and junctions, Figs. 3(a) and 3(b) show a T-branched geometry, and Figs. 3(c) and 3(d) show a cross geometry, obtained by allowing their corresponding sculpted bilayers to reconstruct. These hollow structures could form the key components of future nanofluidic devices or sub-10-nm circuitry.

In Ref. 8, it is demonstrated that simple rectangular nanoribbons sculpted from bilayer graphene can also reconstruct to 


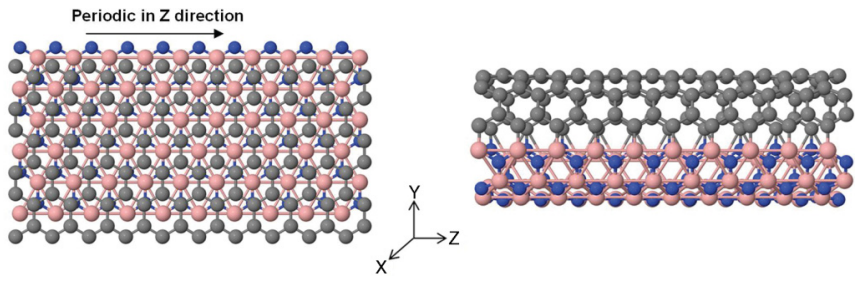

FIG. 4. (Color online) Left: A supercell of heterobilayer nanoribbons of boron nitride/graphene which contains 240 atoms. Right: A hetero-CNT obtained by relaxing the structure on the left.

form CNTs with a predetermined chirality. At first sight, the spontaneous formation of CNTs from bilayer graphene nanoribbons is counterintuitive because this is the reverse of a known process in which CNTs can unzip to form GNRs. ${ }^{18-24}$ However, the experiments demonstrating the latter were performed on relatively wide-diameter CNTs, whereas in Ref. 41, it is noted that the formation of CNTs from free-standing bilayer graphene nanoribbons is energetically favored only for nanoribbons of width less than $3 \mathrm{~nm}$. If the nanoribbons are greater than this width, then they remain flat even though their edges can reconstruct to maximize $s p^{2}$ bonding. Similarly, the global reconstruction leading to the above toroidal sculpturenes and multiconnected structures occurs when the width of the bilayer graphene nanoribbon (BiGNR) is of the order of $3 \mathrm{~nm}$ or less and therefore the inner and outer ECs are in close proximity. Wider BiGNRs would possess ECs, but the cross sections of their interiors would assume a "dog-bone" shape.

Sculpturenes can also be formed from different materials. As an example, Fig. 4 shows a heterobilayer formed from a graphene sheet, $A B$ stacked onto a boron nitride sheet. After sculpting the bilayer to form a nanoribbon and allowing the ribbon to relax, the sculpturene on the right is formed, which comprises a heteronanotube with one half formed from carbon and the other from boron nitride. Heterotoroidal sculpturenes can also be formed in this way.

In summary, we have demonstrated that sculpted bilayer graphene and heterobilayers can reconstruct spontaneously to form unique forms of $s p^{2}$-bonded molecular structures, which have dimensions comparable to those of conventional fullerenes and nanotubes. The toroidal sculpturene of Figs. 1(b) and 1(c) is of the order of 100 times smaller than ring structures reported elsewhere in the literature, which are either formed accidentally ${ }^{47,48}$ or by etching CNTs to form loops with diameters of the order of 0.5 microns. ${ }^{49}$ Furthermore, the sculpturene of Figs. 1(e) and 1(f) has an unprecedented connection $k$ that is greater than 3 . During recent years, there have been several studies of edge morphology in both $A B$ - stacked and $A A$-stacked graphene layers, and rapid progress is being made in achieving control at an atomistic level. ${ }^{33,50-52}$ Even without such control, the sculpting of bilayers to create new topologies is robust because topological invariants are independent of the detailed atomic structure. The presence of a hydrophobic pocket in the center of the torus is also a robust topological feature, which has potential supramolecular sensing applications, particularly when such a structure is embedded in nanocircuitry.

By sculpting bilayer graphene, we have demonstrated that multiterminal sculpturenes with nontrivial shapes can be formed deterministically, including $\mathrm{T}$ junctions, crosses, and toruses. The method can also be used to produce heteronanotubes. As an example, we examined the spontaneous formation of nanotubes obtained by sculpting a heterobilayer from a monolayer of graphene on top of a single layer of boron nitride and find that the resulting nanotubes consist of a half cylinder of carbon joined to a half cylinder of boron nitride. Clearly, the method can be generalized to create heterobilayers formed from other combinations of layered materials and heterosculpturenes with nontrivial topologies. Many other examples of sculpturenes are possible.

In Ref. 41, it is noted that for free-standing GNRs, global reconstruction occurs only when ECs are separated by an order of $3 \mathrm{~nm}$ or less. For the future, it will be of interest to develop methods for promoting the global reconstruction of wider GNRs. One such method is likely to involve chemically coating the GNRs during or after sculpting, since this is known to promote the rolling of flat graphene into curved surfaces. ${ }^{8}$ Even without such assistance, the size of sculpturenes is not restricted to the nanoscale and is limited only by the size of the initial bilayer and the ability to cut the bilayer. For example, if the cross-sectional widths of the six sections forming the hexagon in Fig. 1(a) are held constant, but the size of the structure is increased by increasing the length of the linear strips between the corners, then a larger toroidal sculpturene is formed, with fullerenelike chambers at the corners connected by CNTs. Networks of fullerenelike chambers connected by CNTs could form a basis for nanofluidic devices in which molecules can flow between the chambers via CNT interconnects of the kind shown in Figs. 2 and 3. At present, STM lithography ${ }^{11}$ can be used for the top-down sculpting of GNRs and other shapes with length scales less than $3 \mathrm{~nm}$. Other methods are continuously evolving, including techniques for the cutting of graphene using nanoparticles, ${ }^{12,13,53-55}$ although these are typically limited to shapes with dimensions greater than $10 \mathrm{~nm}$.

This work is supported by the European Union Marie-Curie Networks FUNMOLS and NANOCTM, the UK EPSRC, and the Iraqi Ministry of Higher Education and Scientific Research.

\footnotetext{
*c.lambert@lancaster.ac.uk

${ }^{1}$ M. S. Dresselhaus, G. Dresselhaus, and P. C. Eklund, Science of Fullerenes and Carbon Nanotubes (Academic, Boston, 1996).

${ }^{2}$ The Chemical Physics of Fullerenes 10 Years Later, edited by W. Andreoni (Kluwer, Dordrecht, 1996).
}

${ }^{3}$ Science and Technology of Fullerene Materials, edited by P. Bernier, D. S. Bethune, L. J. Chiang, T. W. Ebbesen, R. M. Metzger, and J. W. Mintmire, MRS Symposium Proceedings (Materials Research Society, Pittsburgh, 1995), Vol. 359.

${ }^{4}$ For a recent review, see Y. Lan, Y. Wang, and Z. F. Ren, Adv. Phys. 60, 553 (2011). 
${ }^{5}$ T. W. Ebbesen and P. M. Ajayan, Nature (London) 358, 220 (1992).

${ }^{6}$ A. Thess et al., Science 273, 483 (1996).

${ }^{7}$ W. Li et al., Science 274, 1701 (1996).

${ }^{8}$ D. Yu and F. Liu, Nano Lett. 7, 3046 (2007).

${ }^{9}$ M. Y. Han, B. Ozyilmaz, Y. Zhang, and P. Kim, Phys. Rev. Lett. 98, 206805 (2007).

${ }^{10}$ Z. Chen, Y.-M. Lin, M. J. Rooks, and Ph. Avouris, Physica E 40, 228 (2007).

${ }^{11}$ L. Tapaszto, G. Dobrik, P. Lambin, and L. P. Biro, Nature Nanotechnol. 3, 397 (2008).

${ }^{12}$ S. S. Datta, D. R. Strachan, S. M. Khamis, and A. T. C. Johnson, Nano Lett. 8, 1912 (2008).

${ }^{13}$ L. J. Ci et al., Nano Res. 1, 116 (2008).

${ }^{14}$ L. C. Campos, V. R. Manfrinato, J. D. Sanchez-Yamagishi, J. Kong, and P. Jarillo-Herrero, Nano Lett. 9, 2600 (2009).

${ }^{15}$ J. Campos-Degado et al., Nano Lett. 8, 2773 (2008).

${ }^{16}$ X. Li et al., Science 319, 1229 (2008).

${ }^{17}$ Z. S. Wu et al., Nano Res. 3, 16 (2010).

${ }^{18}$ L. Y. Jiao, L. Zhang, X. R. Wang, G. Diankov, and H. Dai, Nature (London) 458, 877 (2009).

${ }^{19}$ D. V. Kosynkin et al., Nature (London) 458, 872 (2009).

${ }^{20}$ Z. X. Hang, Z. Z. Sun, J. Yao, D. V. Kosynkin, and J. M. Tour, J. Am. Chem. Soc. 131, 13460 (2009).

${ }^{21}$ A. G. Cano-Marquez et al., Nano Lett. 9, 1527 (2009).

${ }^{22}$ A. L. Elias et al., Nano Lett. 10, 366 (2010).

${ }^{23}$ W. S. Kim et al., Appl. Phys. Lett. 95, 083103 (2009).

${ }^{24}$ J. Jiao, X. Wang, G. Diankov, H. Wang, and H. Dai, Nature Nanotechnol. 5, 321 (2010).

${ }^{25}$ J. Cai et al., Nature (London) 466, 470 (2010).

${ }^{26}$ L. Tapaszto, G. Dobrik, P. Lambin, and L. Biro, Nature Nanotechnol. 3, 397 (2008).

${ }^{27}$ F. Schwierz, Nature Nanotechnol. 5, 487 (2010).

${ }^{28}$ B. Obradovic et al., Appl. Phys. Lett. 88, 141102 (2006).

${ }^{29}$ T. Fang, A. Konar, H. Xing, and D. Jena, Phys. Rev. B 78, 205403 (2008).

${ }^{30}$ M. Bresciani, P. Palestri, D. Esseni, and L. Selmi, in Proceedings of the ESSDERC, 2009 (IEEE, Budapest, 2009), pp. 480-483.

${ }^{31}$ A. Betti, G. Fiori, G. Iannaconne, and Y. Mao, in IEDM Tech. Dig., 2009 (IEEE, Baltimore, 2009), pp. 897-900.
${ }^{32}$ X. Wang, Y. Ouyang, X. Li, H. Wang, J. Guo, and H. Dai, Phys. Rev. Lett. 100, 206803 (2008).

${ }^{33}$ Z. Liu, K. Suenaga, P. J. F. Harris, and S. Iijima, Phys. Rev. Lett. 102, 015501 (2009).

${ }^{34}$ X. T. Jia et al., J. Vac. Sci. Technol. B 27, 1996 (2009).

${ }^{35}$ International Technology Roadmap for Semiconductors, http:// www.itrs.net/Links/2011ITRS/Home2011.htm (unpublished).

${ }^{36}$ J. Soler, E. Artacho, J. Gale, A. García, J. Junquera, P. Ordejón, and D. J. Sánchez-Portal, J. Phys. Condens. Matter 14, 2745 (2002).

${ }^{37}$ S. F. Boys and F. Bernardi, Mol. Phys. 19, 553 (1970).

${ }^{38}$ I. T. Todorov, W. Smith, K. Trachenko, and M. T. Dove, J. Mater. Chem. 16, 1911 (2006).

${ }^{39}$ S. L. Mayo, B. D. Olafson, and W. A. Goddard III, J. Phys. Chem. 94, 8897 (1990).

${ }^{40}$ D. Hilbert and S. Cohn-Vossen, Anschauliche Geometrie (Springer, Berlin, 1932).

${ }^{41}$ N. Byers and C. N. Yang, Phys. Rev. Lett. 7, 46 (1961).

${ }^{42}$ M. Büttiker, Y. Imry, and R. Landauer, Phys. Lett. A 96, 365 (1983).

${ }^{43}$ C. P. Liu and N. Xu, Physica B 403, 2884 (2008).

${ }^{44}$ H. Bluhm, N. C. Koshnick, J. A. Bert, M. E. Huber, K. A. Moler, Phys. Rev. Lett. 102, 136802 (2009).

${ }^{45}$ A. C. Bleszynski-Jayich et al., Science 326, 272 (2009).

${ }^{46}$ X. Tang, J. Phys. Condens. Matter 23, 105302 (2011).

${ }^{47}$ J. Liu et al., Nature (London) 385, 780 (1997).

${ }^{48}$ M. Ahlskog et al., Chem. Phys. Lett. 300, 202 (1999).

${ }^{49}$ M. Sano, A. Kamino, J. Okamura, and S. Shinkai, Science 293, 1299 (2001).

${ }^{50}$ G. Gao, T. Cagin and W. A. Goddard III, Nanotechnology 9, 184 (1998).

${ }^{51}$ E. Cruz-Silva, A. R. Botello-Mendez, Z. M. Barnett, X. Jia, M. S. Dresselhaus, H. Terrones, M. Terrones, B. G. Sumpter, and V. Meunier, Phys. Rev. Lett. 105, 045501 (2010).

${ }^{52}$ X. Jia, J. Campos-Delgado, M. Terrones, V. Meunier, and M. S. Dresselhaus, Nanoscale 3, 86 (2011).

${ }^{53}$ L. Ci et al., Adv. Mater. 21, 1 (2009).

${ }^{54}$ A. L. Higginbotham et al., ACS Nano 4, 2059 (2010).

${ }^{55}$ L. Y. Jiao et al., Nature Nanotechnol. 5, 321 (2010). 